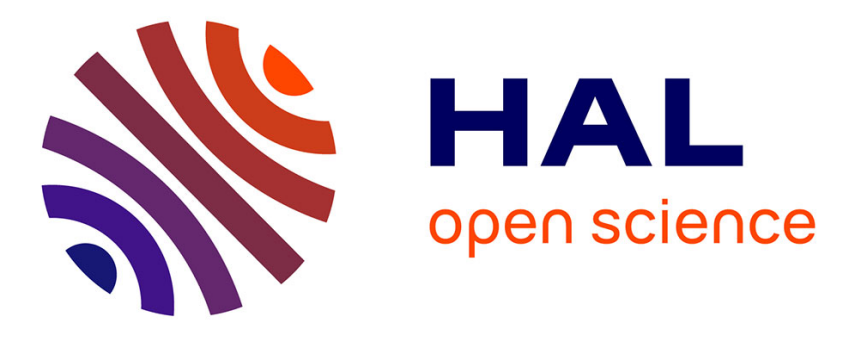

\title{
Grafting odorant binding proteins on diamond bio-MEMS
}

\author{
Raafa Manai, Emmanuel Scorsone, Lionel Rousseau, F. Ghassemi, Maira \\ Possas Abreu, Gaelle Bazin Lissorgues, N. Tremillon, H. Ginisty, Jean-Charles \\ Arnault, E. Tuccori, et al.
}

\section{To cite this version:}

Raafa Manai, Emmanuel Scorsone, Lionel Rousseau, F. Ghassemi, Maira Possas Abreu, et al.. Grafting odorant binding proteins on diamond bio-MEMS. Biosensors and Bioelectronics, 2014, 60, pp.311-317. 10.1016/j.bios.2014.04.020 . hal-01005176v2

\section{HAL Id: hal-01005176 https://hal.science/hal-01005176v2}

Submitted on 25 Jun 2014

HAL is a multi-disciplinary open access archive for the deposit and dissemination of scientific research documents, whether they are published or not. The documents may come from teaching and research institutions in France or abroad, or from public or private research centers.
L'archive ouverte pluridisciplinaire HAL, est destinée au dépôt et à la diffusion de documents scientifiques de niveau recherche, publiés ou non, émanant des établissements d'enseignement et de recherche français ou étrangers, des laboratoires publics ou privés. 


\section{Grafting odorant binding proteins on diamond bio-MEMS}

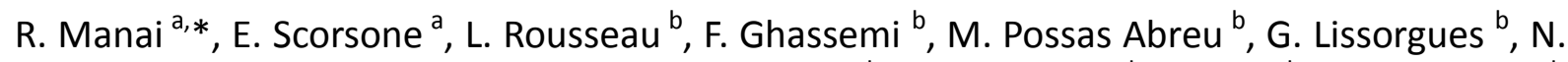
Tremillon ${ }^{c}$, H. Ginisty ${ }^{c}$, J-C. Arnault ${ }^{a}$, E. Tuccori ${ }^{d}$, M. Bernabei ${ }^{d}$, K. Cali ${ }^{d}$, K.C. Persaud ${ }^{d}$, P.Bergonzo ${ }^{a}$

${ }^{\text {a } C E A, ~ L I S T, ~ D i a m o n d ~ S e n s o r ~ L a b o r a t o r y, ~ 91191, ~ G i f-s u r-Y v e t t e, ~ F r a n c e ~}$

${ }^{\mathrm{b}}$ ESIEE-Paris, ESYCOM University Paris-EST, Cité Descartes BP99, 93162 Noisy le Grand Cedex, France

${ }^{\mathrm{d}}$ The University of Manchester, School of Chemical Engineering and Analytical Science, Oxford Road, Manchester, M13 9PL, UK

' GTP Technology, Immeuble Biostep, 436 Rue Pierre et Marie Curie, 31670 Labège, France

* Corresponding author. Tel. : +33169086935; Fax:+33169087819.

E-mail address: raafa.manai@cea.fr (R. Manai)

\section{Abstract:}

Odorant binding proteins (OBPs) are small soluble proteins found in olfactory systems that are capable of binding several types of odorant molecules. Beside, cantilevers based on polycrystalline diamond surfaces are very promising as chemical transducers. Here two methods were investigated for chemically grafting porcine OBPs onto polycrystalline diamond surfaces for the development of biosensors. The first approach results in random orientation of the immobilized proteins over the surface. The second approach based on complexing a histidine-tag located on the protein with nickel allows control of the proteins orientation. These grafting methods were confirmed using electrochemical impedance spectroscopy, fluorescence imaging and X-ray photoelectron spectroscopy analysis. The sensing performances of these OBP modified transducers were assessed. The second grafting method led to typically $20 \%$ more sensitive sensors, as a result of better access of ligands to the proteins active sites and also perhaps a better yield of protein immobilization. This new grafting method appears as highly promising for further investigation of the ligand binding properties of OBPs in general and for the development of non-specific biosensors arrays for artificial olfaction applications.

\section{Keywords:}

Diamond cantilevers

Odorant binding protein

Artificial olfaction

\section{Introduction}

The demand for volatile organic compounds (VOC) detection and identification has increased over the years due to the needs for environmental monitoring, homeland security or medical applications, to name only a few. Amongst the sensing technologies available for VOC detection, biosensors mimicking the way that noses operate to discriminate odorant compounds are highly promising (Sankaran et al., 2012).

Odorant binding proteins (OBPs) are important proteins present in many vertebrates and insect olfactory systems. They are small proteins, involved in the perireceptor events associated with olfaction (Dal Monte et al., 1993; Spinelli et al., 1998; Pelosi et al., 2001; Briand et al., 2002). They were initially discovered by Pelosi and co-workers in the nasal olfactory mucosa of bovines and other mammals as a soluble protein able of binding 2-isobutyl-3-methoxypyrazine (IBMP), an odorant 
molecule with an extremely low detection threshold (Pelosi et al., 1982). Vertebrate OBPs are about $17-20 \mathrm{kDa}$; they belong to the superfamily of lipocalins and are mainly organized in a $\beta$-barrel structure, with a calyx-shaped cavity, made up of eight antiparallel $\beta$-sheets with a short segment of $\alpha$ - helix at the C-terminus (Flower, 1999). The ligand binding site is located in the core of the $\beta$-barrel, as clearly demonstrated by the use of a selenium-containing ligand, 2-amino-4-butyl-5propylselenazole (Napolitano et al., 1992). Their ability to bind diverse odorants, together with their high conformational stability are attractive characteristics for the design of label free biosensors in particular for the detection of small organic molecules (Garibotti et al., 1997; Vincent et al., 2000; Persaud et al., 1996; Pelosi et al., 2013).

Label-free biosensors are generally based on optical, electrochemical or gravimetric transduction methods. Transducers based on microcantilever technology are attractive for ligand binding proteins based biosensors that do not involve electrochemical processes, because they can offer high sensitivity, they can be miniaturized and are suitable for parallelization into arrays. Microcantilevers have recently opened new perspectives for instance for the detection of different toxic metal ions, volatile organics, explosives, etc. (Baller et al., 2000 ; Lavrik et al., 2004 ; Goeders et al., 2008; Boisen et al., 2011; Hwang et al., 2011 ; Spitzer et al., 2012).

We report on the use of poly-crystalline diamond micro-cantilevers combined with OBP receptors for the detection of small volatile organic molecules. Diamond is highly desirable for the design of microcantilevers because it exhibits exceptional mechanical properties, such as a very high Young's modulus in the order of $1000 \mathrm{GPa}$ (Bongrain et al., 2009) as well as an excellent hardness and robustness. Moreover, its carbon nature offers wide opportunities for stable grafting of wide a range of bio-receptors via covalent C-C binding (Härtl et al., 2004; Agnès et al., 2010). The extreme sensitivity of diamond microcantilevers to surface stress variations upon chemical or biochemical interactions occurring on their sensitive surface was demonstrated recently (Bongrain et al., 2011). In this case, the high Young's modulus of diamond allowed the cantilevers to be operated in the resonant regime in the liquid phase while retaining a reasonably high quality factor ( $Q$-factor).

In the present study, the immobilization of OBPs on diamond surfaces is investigated. Thus, we explore immobilization of a wild-type OBP (wtOBPpig) as well as a mutant OBP (m6hisOBPpig), both from pig, on diamond microcantilevers using two different original grafting strategies: (i) hexanoic acid (ha) covalently attached to hydrogen-terminated diamond surfaces followed by 1-ethyl-3-[3dimethylaminopropyl]-carbodiimidehydrochloride/sulfo-N-hydroxysuccinimide (EDC/NHS) coupling with OBP and (ii) a method based on diamond modified with nitrolotriacetic acid nickel complex $\left(\mathrm{NTA}_{\mathrm{Ni}}{ }^{2+}\right.$ ) able to bind specifically to histidine-tagged proteins. Immobilization of OBPs was validated using electrical impedance spectroscopy (EIS), fluorescence methods and X-ray photoelectron spectroscopy (XPS). The first immobilization strategy results in random orientation of the proteins over the sensor surface whereas the latter approach enables orientation of the proteins due to the controlled position of the histidine tag attached to the $\mathrm{N}$-terminal of the proteins. The sensing performances of the two resulting sensors are compared for IBMP, a reference compound commonly used for the characterization of OBPs affinity constants, as well as for 2,4-dinitrotoluene (2,4-DNT), used as a surrogate for the explosive trinitrotoluene (TNT).

\section{Material and methods}

\subsection{Boron Doped Diamond Electrodes (BDD) fabrication}

BDD films with a boron concentration typically of $2 \times 10^{21}$ at $\cdot \mathrm{cm}^{-3}$ were synthesized by microwave plasma enhanced chemical vapor deposition (MPECVD) over a p-Si (100) substrate using a SEKI 
Technotron Corp. AX6500X reactor with a gas mixture of methane, hydrogen and trimethylboron at typically $800{ }^{\circ} \mathrm{C}$ with a pressure of $25 \mathrm{mbar}$ and a microwave power of $2600 \mathrm{~W}$. After growth the electrodes are naturally hydrogen terminated. Some of the electrodes used for control experiment were oxidized under ultraviolet (UV) light/ozone exposure using an excimer UV lamp (Heraeus) generating intensive radiation at $172 \mathrm{~nm}$.

\subsection{Diamond Microcantilever fabrication}

Diamond microcantilevers were fabricated following a process previously described (Bongrain et al., 2009). In brief, silicon moulds prepared by deep reactive ion etching over 4 inches wafers were seeded selectively with diamond nanoparticles using standard clean room patterning approaches. Then, the cantilevers were grown by MPECVD inside the moulds and finally release by dry etching. Cantilever dimensions were $210 \mu \mathrm{m} \times 140 \mu \mathrm{m}$. Before any grafting procedure the diamond cantilever surfaces were re-hydrogenated at $700^{\circ} \mathrm{C}$ in hydrogen plasma. The cantilevers geometry led to a resonance frequency of $145000+/-9900 \mathrm{~Hz}$ and a $\mathrm{Q}$-factor in the order of 600 in air and in the order of 100 in liquid. An SEM image of a typical cantilever used in this study is shown in Fig. S1 in supplementary file.

\subsection{OBPs preparation}

OBPs preparation is reported in detail in supplementary data.

\subsection{First method (1) of OBPs immobilization}

All chemicals used for OBP grafting were purchased from Sigma-Aldrich, France. The first method of OBPs immobilization (Fig. 1(a)) consisted of grafting in a first step a hexanoic acid radical covalently onto hydrogen terminated diamond surface using a process described elsewhere in the case of aminated biotin and ferrocene (Agnès et al., 2010; Bongrain et al., 2011). This covalent immobilization of aliphatic primary amines on hydrogen terminated diamond has been explained by Ruffinato (2010) and co-workers as being a nucleophile substitution involving the formation of carbanion at the diamond surface in the presence of $\mathrm{OH}^{-}$ions in solution. The reaction of the protonated amine with the labile carbanion results in the release of ammonia and C-C covalent grafting of the aliphatic compound over the diamond surface (Ruffinato, 2012). Thus aminocaproic acid (aca) solution at a concentration of $1 \mathrm{mM}$ was prepared by dissolving the adequate amount of aca in a $0.2 \mathrm{M}$ phosphate buffer. The hexanoic acid radical was grafted over the diamond surface by dipping the hydrogen terminated diamond substrates (electrode or cantilever) in the aca solution for $10 \mathrm{~min}$ followed by thorough rinsing in deionised (DI) water. Next the immobilized carboxylic acid was activated with EDC/NHS (30 mM/ $60 \mathrm{mM}$ ) treatment for 2 hours (Fischer, 2010). This protocol enabled subsequent binding to the wtOBPpig protein. Here the substrate was exposed to wtOBPpig solution $\left(0.95 \mathrm{mg} / \mathrm{mL}\right.$ in $50 \mathrm{mM} \mathrm{NaHCO}_{3}$ at $\left.\mathrm{pH}=8.00\right)$ for two hours. Finally the substrates were rinsed thoroughly with DI water and stored in phosphate buffered saline (PBS) solution.

\subsection{Second method (2) of OBPs immobilization}

A second original approach (Fig. 1(b)) was investigated in an attempt to control the orientation of the proteins immobilized on the surface. Here the method consisted of immobilizing a nitriloacetic acid group from $1 \mathrm{mM}$ solution of $\mathrm{N \alpha}, \mathrm{N \alpha}$-Bis-(carboxymethyl)-L-lysine hydrate( $\geq 97.0 \%$ ) (NTA) onto the diamond surface using the same process used for immobilization of aca. Subsequently, the diamond surface was exposed to $1 \mathrm{mM}$ Nickel(II) chloride hexahydrate (99.99\%) solution for $2 \mathrm{~h}$ at room 
temperature. The resulting NTA $\cdot \mathrm{Ni}^{2+}$ complex was formed thus enabling the OBP immobilization. NTA has the ability to bind to Nickel (II), which complex has a high affinity with histidine groups (Johnson et al., 2004; Blankespoor et al., 2005; Brellier et al., 2009). Hence m6hisOBPpig were finally immobilized by exposing the Ni-NTA terminated diamond surfaces to m6hisOBPpig solution (1.0 $\mathrm{mg} / \mathrm{mL}$ in $20 \mathrm{mM}$ PBS, $\mathrm{pH}=8.00$ ) for 2 hours. Again the substrates were rinsed thoroughly with $\mathrm{DI}$ water and stored in PBS solution.

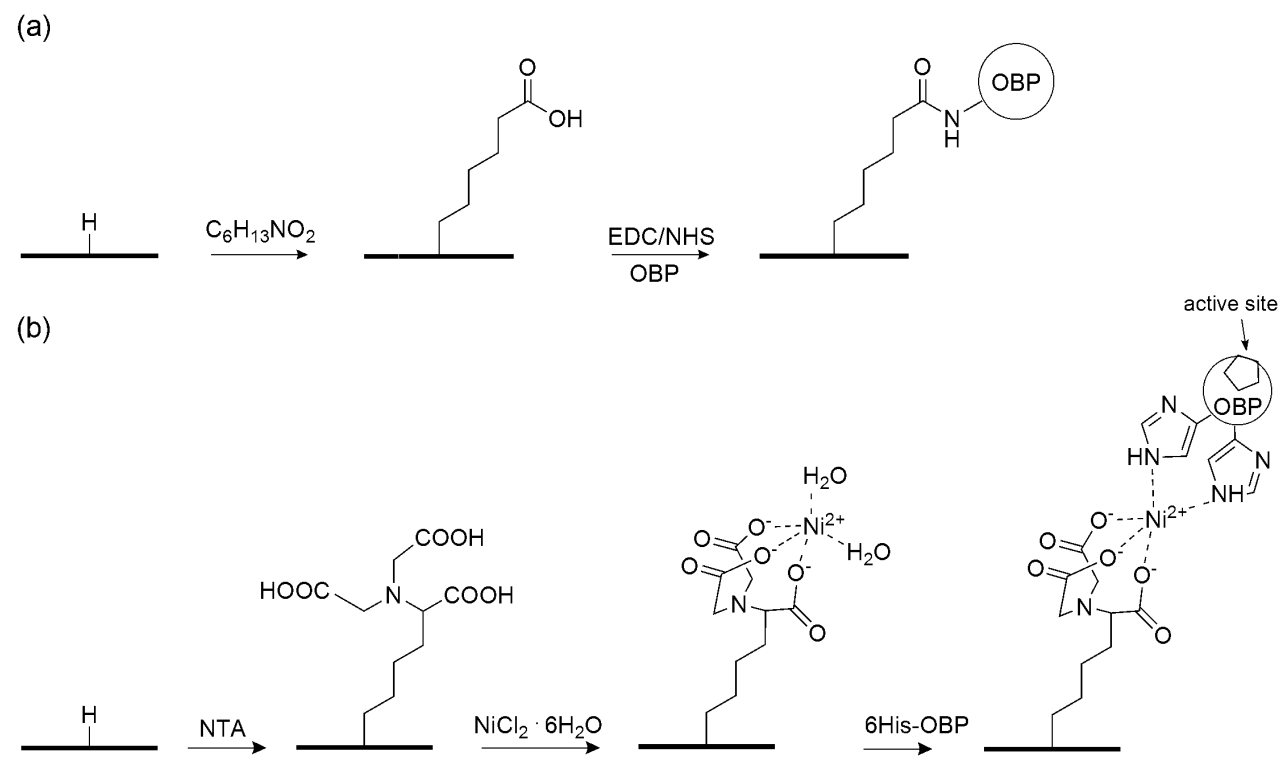

Fig. 1. Summary of OBP immobilization on hydrogenated diamond surfaces using grafting method (1), (a) and using grafting method (2), (b).

\subsection{EIS measurements}

In order to characterize the surface coverage of OBP onto the diamond surface, EIS measurements were performed. The electrochemical characterizations were carried out in a 3 electrodes setup where BDD is the working electrode, and platinum wires both the counter and pseudo-reference electrodes, respectively. Ultrapure DI water (Millipore Direct Q3) was used to make all the solutions. EIS was carried out in equimolar (1 mM) potassium ferricyanide(III)/ potassium hexacyanoferrate(II) trihydrate (Acros Organics) dissolved in $0.5 \mathrm{M}$ potassium chloride solution (Acros Organics). The specific transfer resistances (Rt) were experimentally determined before and after each immobilization step by EIS over a frequency range of $50 \mathrm{kHz}-10 \mathrm{mHz}$ with logarithmic point spacing and potential amplitude of $0.01 \mathrm{~V}$ root-mean-square $(\mathrm{rms})$ while the BDD electrode was maintained at open circuit potential. EIS spectra were recorded after each grafting step for both immobilization procedures, starting from the hydrogen terminated diamond electrode surface to OBPs grafted surface. Nyquist plots were also recorded with oxidized diamond electrodes, used as negative control since in the experimental conditions used the amine terminated primary linker (aca or NTA) can only be immobilized on hydrogen terminated surfaces (Agnès et al., 2010). Hence OBPs can theoretically be immobilized only on such surfaces that contain the immobilized linker.

\subsection{Fluorescence imaging}

Further confirmation of protein immobilization was obtained by fluorescence imaging. For this purpose, two identical samples were prepared as follows. The hydrogen-terminated surface was 
patterned by conventional photolithography using a process similar to that described elsewhere (Härtl et al., 2004). Since attachment of hexanoic acid or NTA radicals via the amino-group is only possible on hydrogen-terminated surfaces, the purpose of this experiment was to demonstrate that the proteins are immobilized indeed only where the diamond surface was originally hydrogenated and therefore where a linker molecule has been attached. It verifies also that no non-specific adsorption of the proteins occurs over the control (oxidized) areas. Thus, a photosensitive resin was deposited onto hydrogen-terminated diamond substrate and patterned ( $50 \mu \mathrm{m} \times 50 \mu \mathrm{m}$ spots) by photolithography. The samples was then oxidized in the unprotected areas under oxygen plasma ( 2 mbar oxygen, $50 \mathrm{~W}, 30 \mathrm{~s}$ ) in a reactive ion etching equipment. The resin was then removed and the grafting procedure applied on the whole sample. After the last step of OBPs grafting, a first sample was immersed in a fluorescein solution then rinsed vigorously with DI water. Fluorescein has the ability to bind the OBP thus offering the possibility to visualize under UV excitation the presence of OBPs on the diamond surface. The same experiment was done two months later with the second sample in order to verify whether the OBP was still present and active over the surface. Fluorescence imaging was also used to verify the presence of OBPs on cantilevers after the immobilization step as well as after several exposures to the target chemical. Fluorescence microscopy images were recorded at $\lambda_{\text {exc }}=550 \mathrm{~nm}$ and $\lambda_{\mathrm{em}}=570 \mathrm{~nm}$.

\subsection{XPS surface analysis}

XPS analyses were used in this study to characterize the NTA-Ni grafting on hydrogenated diamond surface. The methods and experimental conditions used for XPS surface analysis are reported in detail in Supplementary data.

\subsection{Cantilever resonance frequency measurements}

The dynamic response (resonance frequency shift) of the biosensors was achieved by Laser Doppler interferometry using a Polytec setup consisting of an interferometer (OFV534) and a vibrometer controller (OFV5000), coupled to a network analyzer. The operation principle of this equipment has been described elsewhere (Bongrain et al., 2011). This setup can detect vertical oscillations in the picometer range, while cantilevers excited by the piezoelectric cell exhibit amplitudes between tens to one hundred nanometers at their resonance frequency.

The micro-cantilevers were mounted in a liquid cell with a constant volume $(200 \mu \mathrm{L})$ underneath which an external piezo-electric vibrator was placed to allow structures actuation through acoustic waves. A stabilization step was necessary when the cantilever environment changed from air to liquid. This stabilization time is due to temperature stabilization in the liquid cell, adsorption of dissolved salts from the buffer solution on the cantilever surface (Godin et al., 2010) and surface stress stabilization at the cantilever-liquid interface. Exposure to the different test solutions was carried out as follows. $50 \mu \mathrm{L}$ of the blank solution was pipetted out from the cell and replaced by the same volume of either IBMP solution or 2,4-DNT solution so that the cantilevers remained immerged throughout all experiments. Gentle stirring was carried out following analyte addition to ensure homogeneous concentration during measurements. This did not affect the measurements since the kinetics involved through binding with OBPs are typically in the order of several minutes. The resonance frequency shift measurements were recorded with a sampling time of $20 \mathrm{~s}$.

\section{Results and discussion}

\subsection{OBP grafting characterization}




\subsubsection{Electrical Impedance spectroscopy}

Fig. 2. (a) Typical impedance spectra of (black) blank hydrogenated diamond surface, (red) Hterminated diamond surface after immobilizing hexanoic acid radical, (blue) $\mathrm{H}$-terminated diamond surface after immobilizing wtOBPpig (following EDC/NHS treatment on hexanoic acid. (b) Typical impedance spectra of (black) blank oxidized diamond surface, (red) oxidized diamond surface after an attempt to immobilize hexanoic acid radical, (blue) diamond surface after an attempt to immobilize wtOBPpig and in insert a zoom-in of impedance spectra. (c) Transfer Resistance evolution for oxidized and hydrogen-terminated boron doped diamond electrodes measured after each immobilization step using grafting method (1) and using grafting method (2) with relative standard deviation (RSD \%) reported above each errors bar.
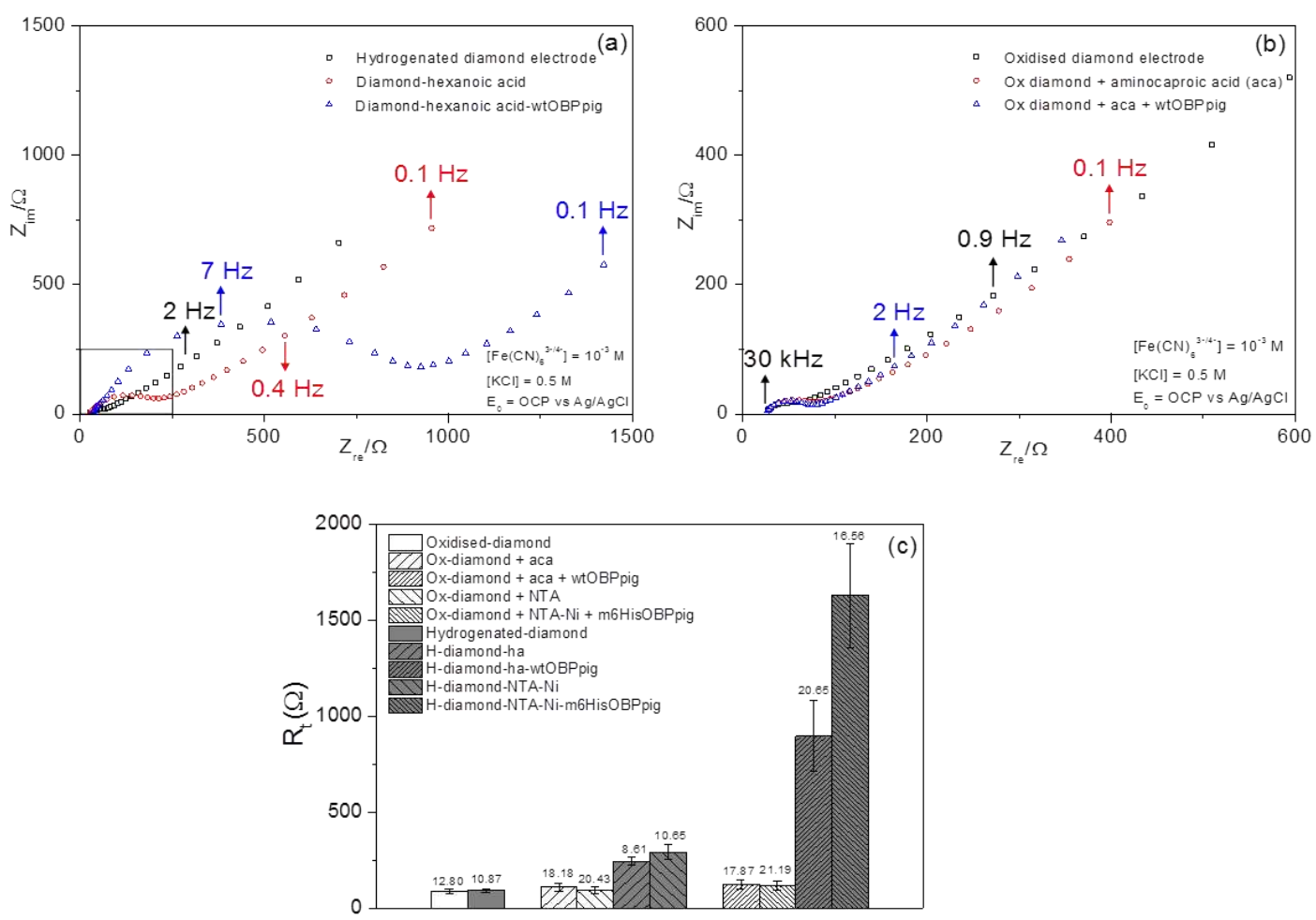

Fig. 2 (a) shows typical Nyquist plots recorded on a bare hydrogen terminated electrode as well as on the same electrode following immobilization of aca and subsequently wtOBPpig by EDC/NHS coupling. The same plot is presented in Fig. 2 (b) for the same immobilization attempt carried out onto an oxidized BDD electrode. The impedance measurements in this case can be analyzed by fitting the impedance spectra to the Randles equivalent circuit (Wang et al., 2004). Here the intercept of the observed semi-circle with the $Z_{R E}$ axis at high frequency gives the value of the solution resistance (Rs), whereas extrapolation of the semi-circle to low frequencies yields an intercept corresponding to $\mathrm{Rs}+\mathrm{Rt}$, where Rt is the charge-transfer resistance. It can be seen clearly that in the case of the hydrogen-terminated electrode, Rt increases slightly from the blank hydrogen terminated surface (black curve) to the aca grafted sample (red curve). Then a further increase, much larger this time, is observed with the same electrode upon OBP immobilization. Electron transfer for $\mathrm{Fe}(\mathrm{CN})_{6}^{-3 / 4}$ proceeds by a inner-sphere transfer mechanism and the electrode kinetics is highly sensitive to the 
electrode surface conditions. Thus the presence of immobilized organic species over the electrode surface causes a significant decrease in the electron transfer rate and therefore an increase in Rt. In contrast, when the same experiment is performed using an oxidized surface, virtually no change in Rt resistance is observed after the different grafting steps. This suggests that hexanoic acid groups and subsequently OBPs are indeed grafted only on the hydrogen terminated diamond surface as expected but not on the oxidized surface.

Fig. 2 (c) summarizes the Rt values extracted from the Randle's model for both electrodes and for both grafting method. In the case of the hydrogen terminated electrode, Rt is increased from typically $78 \Omega$ to $896 \Omega$ from the blank electrode to the OBP grafted electrode using method (1), respectively.

The results were very similar and immobilization was also successful only on the hydrogen terminated electrode with the grafting method (2), based on NTA grafting followed by the NTA-Ni ${ }^{2+}$ complex formation which enables the m6hisOBPpig immobilization via histidine group. Here the Rt values increased from typically $72 \Omega$ to $1630 \Omega$ from the bare diamond electrode to the OBP grafted electrode, respectively. The significantly higher Rt value obtained for the OBP grafted electrode using method (2) tends to indicate that this latter method is more efficient and more OBPs are present over the electrode surface.

After immobilization of m6hisOBPpig on the electrode surface using method (2), the surface was exposed to imidazole ( $200 \mathrm{mM}$ in PBS at $\mathrm{pH}=7.4$ ). Imidazole has the ability to binds competitively NTA against $\mathrm{Ni}^{2+}$, which has for effect to release OBPs off the surface. This experiment was carried out in order to verify that OBPs were indeed linked to nickel rather than being non-specifically adsorbed onto the surface. Fig. S2 (see Supplementary data) shows that after exposure to imidazole solution, the transfer resistance Rt indeed is very significantly reduced from typically $1677 \Omega$ to 394 $\Omega$, most presumably as a result of OBPs detachment. This tends to give further evidence that OBPs are indeed attached to the diamond surface via complexation of nickel.

Fig. S3 (see Supplementary data) reports the assessment of OBP immobilization kinetic by recording the evolution of Rt upon exposure of NTA-Ni modified electrode to m6hisOBPpig solution at a concentration of $1.0 \mathrm{mg} / \mathrm{mL}$. The resulting curve is showing that a plateau is achieved after typically 200 minutes $\left(T_{90 \%}=140 \mathrm{~min}\right)$.

\subsubsection{Fluorescence imaging}

OBP immobilization was also evidenced by fluorescence imaging using the protocol described in section 2.4. Fig. 3 shows a resulting fluorescence microscopic image of wtOBPpig immobilized on hydrogen-terminated diamond patterns. This experiment confirmed the presence of OBPs only on the region where the diamond was originally hydrogenated. The absence of any fluorescence signal on the oxidized area confirms also that no significant non-specific OBP adsorption occurs outside the pattern. The same experiment was repeated with the second grafting method and led to the same result. No significant difference in fluorescence was observed when the process was repeated using a second sample that was stored in air for two month after grafting the OBPs. 


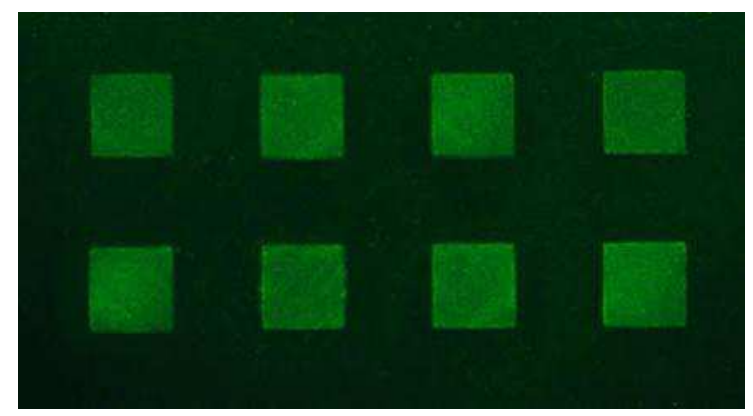

Fig. 3. Fluorescence microscopy picture observed at $\lambda \mathrm{exc}=550 \mathrm{~nm}$ and $\lambda \mathrm{em}=570 \mathrm{~nm}$ of diamond patterned by oxidation and functionalized with OBP immobilization after the immersion in a fluorescein solution.

\subsubsection{Characterization of the NTA-Ni functionalized diamond: XPS analysis}

XPS analyses were used to investigate the binding state of nickel on hydrogenated diamond surface after modification with NTA-Ni complexes. According to XPS spectrum, the presence of nickel on NTA-Ni grafted on diamond surface sample was confirmed. The corresponding atomic nickel concentration is 2.7 at.\%. Fig. 4 shows the high resolution Ni $2 p$ XPS spectrum exhibiting the doublet components of $\mathrm{Ni} 2 \mathrm{p}_{3 / 2}$ and $\mathrm{Ni} 2 \mathrm{p}_{1 / 2}$ located at $856.8 \mathrm{eV}\left(\mathrm{Ni} 2 \mathrm{p}_{3 / 2}\right.$ ) and $874.6 \mathrm{eV}\left(\mathrm{Ni} 2 \mathrm{p}_{1 / 2}\right.$ ), respectively. The $\mathrm{Ni} 2 \mathrm{p}$ spectrum also reveals the presence of multiplet structures and shake-up satellites. The $\mathrm{Ni} 2 \mathrm{p}$ binding energies are very close to the ones reported for $\mathrm{Ni}_{2} \mathrm{O}_{3}\left(\mathrm{Ni}_{2} 2 \mathrm{p}_{3 / 2}\right.$ at 857.0 $\mathrm{eV}$ ) (Chien et al., 2011) while Ni $2 \mathrm{p}_{3 / 2}$ is located at $853.7 \mathrm{eV}$ for NiO (Biesinger et al., 2009).

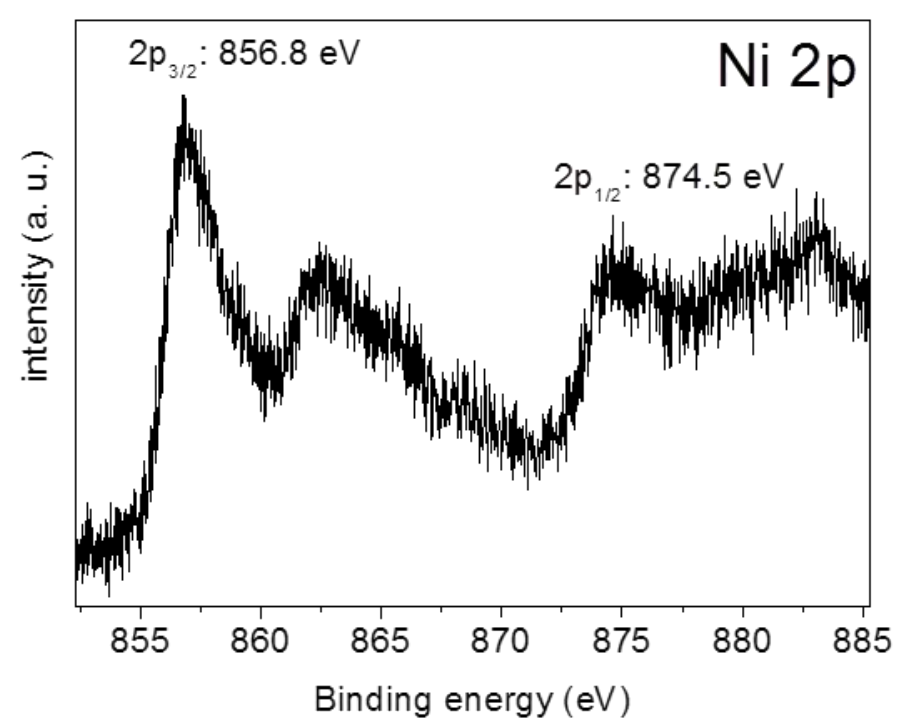

Fig. 4. High resolution Ni $2 p$ core level XPS spectrum of NTA-Ni modified hydrogenated diamond surface.

\subsection{Sensor performances}

The detection of IBMP and 2,4-DNT with diamond microelectromechanical systems (MEMS) sensors grafted with m6hisOBPpig was investigated using both methods (1) and (2) as described previously. At start, the resonance frequency of cantilevers was measured in air and then in an aqueous volume of $200 \mu \mathrm{L}$ at $\mathrm{pH}=8$. As expected the resonance frequency strongly decreases from typically $145 \mathrm{kHz}$ to 
approx. $35 \mathrm{kHz}$ when dipped into the buffer solution. This is due to damping resulting from the increase of viscosity of the surrounding liquid medium (Vancura et al., 2008). Then, a volume of $50 \mu \mathrm{L}$ of this solution was replaced by the same volume of either IBMP or 2,4-DNT solutions at various concentrations to cover the concentration range from $60 \mu \mathrm{M}$ to $1.32 \mathrm{mM}$ for IBMP and 2,4-DNT, after dilution. After each subsequent analyte addition, a significant increase of the resonance frequency was observed. The new frequency values were recorded when a plateau was reached. Each value is given for a set of five different cantilevers in order to assess the reproducibility of the entire process. Each data point is the mean value recorded for the five cantilevers. The highest frequency shift recorded was in the order of $300 \mathrm{~Hz}$ in the case of IBMP exposure for a concentration of $1.32 \mathrm{mM}$. The stability of the OBPs on the surface of the cantilever was also explored. Experiments were performed with the same cantilever over 2 month periods with no significant change in sensitivity after 2 month storage in air at room temperature.
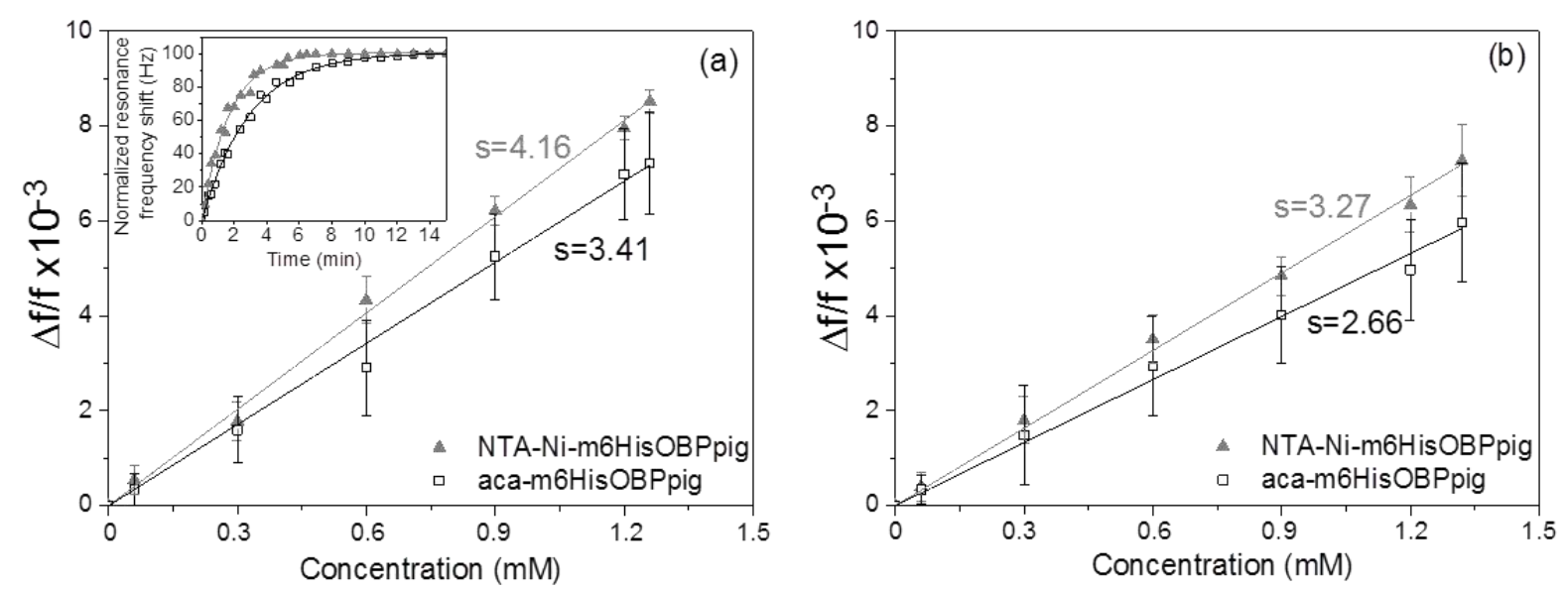

Fig. 5. Concentration response profile for NTA-Ni-m6hisOBPpig and aca-m6hisOBPpig based biosensors (a) upon exposure to different concentrations of IBMP, inset: kinetics response curves upon a specific concentration of IBMP; (b) upon exposure to different concentrations of 2,4-DNT. Errors bars: +/- Standard deviation (SD) calculated from 5 measurements carried out on 5 cantilevers.

RSD for figure 5 are reported in detail in supplementary data in Table S1.

Calibration curves for both biosensors in response to the target analytes is shown in Fig. 5 . In all cases, the sensor response is linear with the analyte concentrations. Table 1 summarizes for each sensor the values of sensitivities, with the time constants at different concentrations of odorant concentrations extracted by fitting a first-order exponential regression on the response curves. The highest sensitivities and response times are achieved for IBMP. This is expected since the affinity constant of porcine OBP for this compound is known to be very high (Pelosi et al ., 1982; Pevsner et al., 1985; Baldaccini et al., 1986; Pevsner et al., 1990; Burova etal., 1999). Nevertheless, the sensitivity to 2,4-DNT is also comparatively high and confirms the fairly high sensitivity of porcine OBP to aromatic compounds as reported by Vincent et al., 2000; Ramoni et al., 2007 and Wei et al., 2008. Furthermore, the sensitivity of the NTA-Ni-m6hisOBPpig sensor to both odorant molecules is significantly higher than the sensitivity of the aca-m6hisOBPpig sensor, here typically $20 \%$ higher for both IBMP and 2,4-DNT.

The sensors modified by NTA-Ni complex linker was found to be generally more sensitive to odorants and more reproducible than the sensors modified with aca-m6HisOBPpig. This could be explained by 
the better access of ligand molecules to the active sites of the proteins and also perhaps a better yield of immobilization of the proteins when using the NTA-NI linker. In both cases the sensitivity toward IBMP and 2,4-DNT was fairly close. This is expected since porcine OBP is relatively nonspecific and has the ability to bind a broad range of molecules. The response of the cantilevers is mainly due to surface stress variations arising from slight conformal changes of the proteins upon binding. Even though those conformal changes are known to be small, a reasonable sensitivity was observed and a linear relationship was found between concentration of ligand and frequency variations. This result suggests that orientating the protein over the surface has a significant effect on the overall sensitivity. Since the active site of the "cage molecule" is facing the outside environment more molecules are likely to bind the receptors. A better yield of the grafting reaction resulting in more OBPs being immobilized over the surface as suggested by a larger increase of Rt upon grafting with method (2) (Fig. 2 (c)) may also contribute to the better sensitivity. Nevertheless, for both IBMP and 2,4-DNT, the time constants for the sensors grafted with method (2) are significantly higher than those measured with method (1), therefore confirming that the active sites of the proteins are probably more easily accessible in this case. Interestingly also, one can notice in table 1 as well as on the error bars in Fig. 5 (a) and (b) that the calculated standard variations are significantly smaller for method (2), indicating that method (2) is more reproducible. In fact, one could easily interpret that when OBPs are randomly oriented over the surface; the number of active sites available varies randomly from sensor to sensor, resulting in the significantly higher standard variations. On the other hand, when all sites are equally oriented, they are more likely to offer similar number of binding sites, thus leading to more reproducible sensors responses, provided that the density of active sites is constant from one sensor to another.

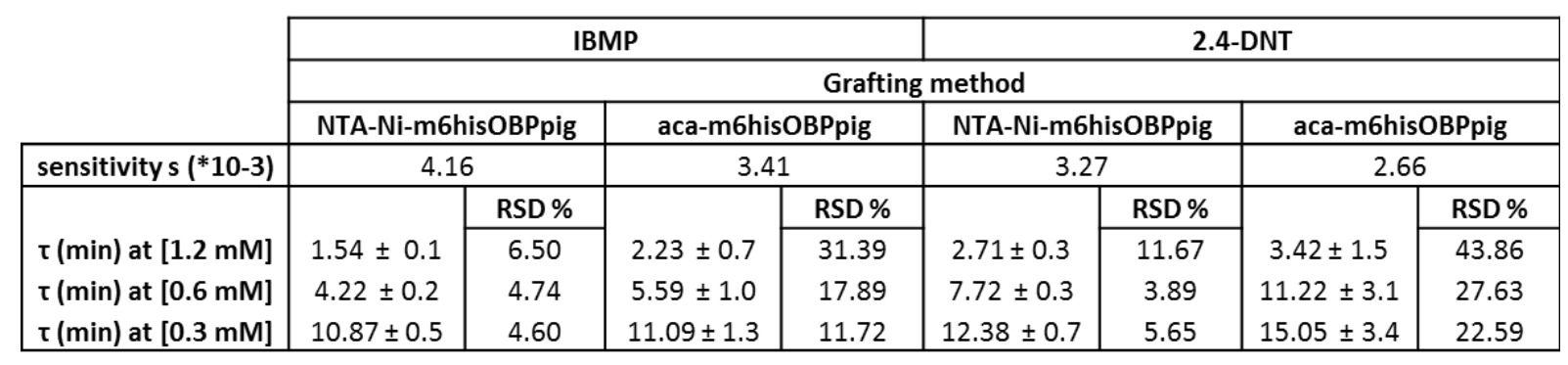

Table 1. Comparison between sensitivities $s$ and $\tau$ for IBMP and 2,4-DNT detection using the two OBP grafting methods with SD and RSD.

In the gas phase, adsorption of molecular species over the transducer surface contributes to increase the cantilever effective mass, which generally results in a decrease of the cantilever resonance frequency. However in liquid media the cantilever mass sensitivity is significantly reduced due to viscous damping and fluid load effects (Chen et al., 1995; Hwang et al., 2006). In the latter case, the resonance frequency of the cantilever may be influenced by hydrodynamic interactions and/or by surface stress variations. Since the binding of the odorant molecules in our case does not involve much charge transfer from or to the OBP, the overall surface charge of the transducer is not likely to be significantly affected by the presence of adsorbed analytes. Moreover, the cantilever response being positive, one can assume that the response is mainly arising from surface stress variations over the cantilever and not from mass loading. A positive response could also be attributed to a loss of mass due to detachment of the OBPs upon conformal changes. It is believed this is not happening because the response was fully reversible after many response cycles. Moreover fluorescence imaging performed on cantilevers after several measurements confirmed the presence of OBPs over 
the surface of the cantilevers. The evolution of the resonance frequency in liquids following mass and bending stiffness variations may be described by equation (1) where $m$ is the effective mass of the cantilever, $E$ is the Young's modulus of the material composing the cantilever (here $980 \mathrm{GPa}$ ), $w, t$ and $I$ are the width, thickness and length of the cantilever, $\Delta k$ and $\Delta m$ are the stiffness and effective mass variations, and $u$ is the mass per unit length applied by the fluid. $u$ can be extracted from the ratio of the measured cantilever resonance frequency in air and in the liquid according to equation (2), where the contribution of the quality factors is neglected (Bongrain et al., 2011).

$$
\begin{gathered}
\frac{\Delta f_{r}^{l i q}}{f_{r}^{l i q}} \cong\left(\sqrt{\left(1+\frac{4 l^{3} \Delta k}{E w t^{3}}\right)} \times \sqrt{\left(1-\frac{\Delta m}{m\left(1+\frac{u l}{m}\right)}\right)}\right)-1 \\
\frac{\Delta f_{r}^{\text {air }}}{f_{r}^{l i q}} \cong\left(\sqrt{\left(1+\frac{u l}{m}\right)}\right)
\end{gathered}
$$

It has been shown that the binding of an odorant molecule can induce local conformational changes in OBP cavities. Such ligand-binding induced conformational changes and folded structure changes generally modify the protein spectral properties and have been studied by UV or Fourier transform infrared spectroscopy. Those conformal changes depend on the type of OBP and the shape of the odorant molecule, as well as on the conformation of the ligand in the OBP cavity (Nespoulous et al., 2004). According to the literature, the porcine OBP lipocalin structure is less affected by the presence of ligands than rat OBPs for instance (Burova et al., 1999). Indeed, very few specific interactions were established inside the porcine OBP cavity between the odorant molecule and the amino acid sidechains, which did not induce large conformational changes (Hajjar et al., 2006). The crystallographic structures of porcine OBPs complexed with odorant molecules revealed that different odorant molecules can interact in the cavity, with alternative conformations, thus revealing the absence of specific interactions and the role of porcine OBPs as non-specific carriers, able to recognize efficiently a large number of molecules belonging to different chemical classes (Vincent et al., 2000, Vincent et al., 2004). This explains probably the relatively small difference in sensitivity between DNT and IBMP. The maximum stiffness variation upon the largest change in resonance frequency obtained during exposure of NTA-NI-m6hisOBPpig modified cantilever to $1.32 \mathrm{mM}$ IBMP calculated using eq (1) was found to be $1.7 \mathrm{~N} . \mathrm{m}^{-1}$. Since the effective stiffness coefficient of this cantilever is fairly high, approximately $\mathrm{k}=100$ according to equation $\mathrm{k}=\mathrm{Ewt}^{3} / 4 \mathrm{I}^{3}$, this corresponds to a stiffness variation of $1.7 \%$. This is relatively weak when compared for instance to the sensitivity of diamond cantilevers grafted with carboxylic acid groups which exhibit a $\Delta \mathrm{k}$ value as high as $6 \%$ upon deprotonation of the acid. This supports the assumption of low conformal changes of the OBP upon ligand-binding and hence the low effect on surface stress. The corresponding sensitivity to IBMP over stiffness variations was found to be $0.163 \mathrm{~Hz} \cdot \mathrm{mN}^{-1} . \mathrm{m}$ for NTA-Ni-m6hisOBPpig and $0.161 \mathrm{~Hz} \cdot \mathrm{mN}^{-1} . \mathrm{m}$ for acam6HisOBPpig cantilevers, respectively. This is almost one order of magnitude below the sensitivity of similar diamond cantilevers to 32base-pair DNA hybridization of probe DNA immobilized over their surface (Bongrain et al, 2010). Indeed in the latter case the sensitivity is expected to be higher since steric effects are more significant due to the large increase in density of DNA strains and the overall charge over the surface. Nevertheless the sensitivity of the diamond cantilevers is sufficient to allow monitoring the ligand binding to $\mathrm{OBP}$ in the $\mu \mathrm{M}$ concentration range. 


\section{Conclusions}

We reported on the grafting of porcine OBP onto diamond microcantilevers using two different chemical approaches enabling either random or oriented immobilization of the proteins over the surface. Surface immobilization of the proteins was validated by impedance spectroscopy, fluorescence imaging and XPS analysis in the case of the oriented method using NTA-Ni complex linker. This grafting technique provided sensors with better sensitivity for the detection of IBMP as well as 2,4-DNT, a simulant for explosive TNT, in the $\mu \mathrm{M}$ concentration range. This is highly promising for further investigating the ligand binding properties of OBPs in general and for the development of reliable non selective biosensors that may be used in vapor sensing applications such as for the detection of explosives. The low specificity of this biosensor will be a certain inconvenience to overcome.

\section{Acknowledgements}

Financial support from the European Commission Research Innovation $-7^{\text {th }}$ Framework Programme (FP7-SECURITY project $n^{\circ} 285203$ ) is gratefully acknowledged.

\section{Appendix A. Supplementary data}

Supplementary data associated with this article can be found in the online version.

\section{References}

Agnès, C., Ruffinatto, S., Delbarre, E., Roget, A., Arnault, J.-C., Omnès, F., Mailley, P., 2010. IOP Conference Series: Materials Science and Engineering 16, 012001.

Baldaccini, N. E., Gagliardo, A., Pelosi, P., Topazzini, A., 1986. Comparative Biochemistry and Physiology Part B: Comparative Biochemistry 84 (3), 249-253.

Baller, M. K., Lang, H. P., Fritz, J., Gerber, C., Gimzewski, J. K., Drechsler, U., Gu, H., 2000. Ultramicroscopy 82, 1-9.

Biesinger, M. C., Payne, B. P., Lau, L. W. M., Gerson, A., Smart, R. S. C., 2009. Surface and Interface Analysis 41 (4), 324-332.

Blankespoor, R., Limoges, B., Schöllhorn, B., Syssa-Magalé, J.-L., Yazidi, D., 2005. Langmuir: the ACS journal of surfaces and colloids 21 (8), 3362-75.

Boisen, A., Dohn, S., Keller, S. S., Schmid, S., Tenje, M., 2011. Reports on Progress in Physics 74 (3), 036101.

Bongrain, A., Scorsone, E., Rousseau, L., Lissorgues, G., Gesset, C., Saada, S., Bergonzo, P., 2009. Journal of Micromechanics and Microengineering 19 (7), 074015.

Bongrain, A., Uetsuka, H., Rousseau, L., Valbin, L., Saada, S., Gesset, C., Bergonzo, P., 2010. Physica Status Solidi (a) 207 (9), 2078-2083.

Bongrain, A., Agnès, C., Rousseau, L., Scorsone, E., Arnault, J.-C., Ruffinatto, S., Bergonzo, P., 2011. Langmuir: the ACS journal of surfaces and colloids 27 (19), 12226-34.

Bongrain, A., Scorsone, E., Rousseau, L., Lissorgues, G., Bergonzo, P., 2011. Sensors and Actuators B: Chemical 154 (2), 142-149.

Bonnot, K., Bernhardt, P., Hassler, D., Baras, C., Comet, M., Keller, V., Spitzer, D., 2010. Analytical chemistry $82(8), 3389-93$

Brellier, M., Barlaam, B., Mioskowski, C., Baati, R., 2009. Chemistry, Weinheim an der Bergstrasse 15 (46), 12689-701. 
Briand, L., Eloit, C., Nespoulous, C., Bezirard, V., Huet, J.C., Henry, C., Blon, F., Trotier, D., Pernollet, J.C., 2002. Biochemistry 41 (23), 7241-7252.

Chen, G. Y., Thundat, T., Wachter, E. A., Warmack, R. J, 1995 Journal of Applied Physics 77 (8), 3618.

Chien, F. S.-S., Wu, Y. T., Lai, G. L., Lai, Y. H., 2011. Applied Physics Letters 98 (15), 153513.

Concina, I., Falasconi, M., Gobbi, E., Bianchi, F., Musci, M., Mattarozzi, M., Sberveglieri, G., 2009.

Food Control 20 (10), 873-880.

Dal Monte, M., Andreini, I., Revoltella, R., Pelosi, P., 1991.Comparative Biochemistry and Physiology Part B: Comparative Biochemistry 99 (2), 445-451.

Dal Monte, M. D., Centini, M., Anselmi, C., Pelosi, P., 1993. Chemical Senses 18 (6), 713-721.

Flower D.R., 1996. Biochem J. 318 (1) ,1-14.

Garibotti, M., Navarrini, A., Pisanelli, A. M., Pelosi, P., 1997. Chemical senses 22 (4), 383-90.

Glatz, R., Bailey-Hill, K., 2011. Progress in neurobiology 93 (2), 270-96.

Godin, M., Tabard-Cossa, V., Miyahara, Y., Monga, T., Williams, P. J., Beaulieu, L. Y., Grutter, P., 2010. Nanotechnology 21 (7), 75501.

Goeders, K. M., Colton, J. S., Bottomley, L. A., 2008. Chemical reviews 108 (2), 522-42.

Fischer, M. J. E., 2010. Surface Plasmon Resonance, Methods in Molecular Biology 627, 55-73.

Hajjar, E., Perahia, D., Débat, H., Nespoulous, C., Robert, C. H., 2006. The Journal of biological chemistry 281 (40), 29929-37.

Härtl, A., Schmich, E., Garrido, J. a, Hernando, J., Catharino, S. C. R., Walter, S., Stutzmann, M., 2004. Nature materials 3 (10), 736-42.

Hwang, K. S., Eom, K., Lee, J. H., Chun, D. W., Cha, B. H., Yoon, D. S., Park, J. H., 2006. Applied Physics Letters 89 (17), 173905.

Hwang, K. S., Lee, M. H., Lee, J., Yeo, W.-S., Lee, J. H., Kim, K.-M., Kim, T. S., 2011. Biosensors bioelectronics 30 (1), 249-54.

Johnson, D. L., Martin, L. L., 2005. Journal of the American Chemical Society 127 (7), 2018-9.

Joo, B.-S., Huh, J.-S., Lee, D.-D., 2007. Sensors and Actuators B: Chemical 121 (1), 47-53.

Lavrik, N. V., Sepaniak, M. J., Datskos, P. G., 2004. Review of Scientific Instruments 75 (7), 2229.

Napolitano, E., Pelosi, P., 1992. Bioorganic Medicinal Chemistry Letters 2 (12), 1603-1606.

Nespoulous, C., 2004. Chemical Senses 29 (3), 189-198.

Pelosi, P., Baldaccini, N. E., Pisanelli, a M., 1982. The Biochemical journal 201 (1), 245-8.

Pelosi, P., 2001. Cellular and Molecular Life Sciences 58, 503-509.

Pelosi, P., Mastrogiacomo, R., lovinella, I., Tuccori, E., Persaud, K. C., 2013. Applied microbiology and biotechnology.

Persaud, K. C., Khaffaf, S. M., Payne, J. S., Pisanelli, A. M., Lee, D.-H., Byun, H.-G., 1996. Sensors and Actuators B: Chemical 36 (1), 267-273.

Pevsner, J., Trifiletti, R. R., Strittmatter, S. M., Snyder, S. H., 1985. Proceedings of the National Academy of Sciences of the United States of America 82 (9), 3050-4.

Pevsner, J., Hou, V., Snowman, A. M., Snowman, M., Snyder, H., 1990. Journal of biological chemistry 265 (11), 6118-6125

Ramoni, R., Bellucci, S., Grycznyski, I., Grycznyski, Z., Grolli, S., Staiano, M., D’Auria, S., 2007. Journal of Physics: Condensed Matter 19 (39), 395012.

Ruffinato, S., 2012. Thesis, Université de Grenoble.

Sankaran, S., Khot, L. R., Panigrahi, S., 2012. Sensors and Actuators B: Chemical 171-172, 1-17.

Sivashanmugam, A., Murray, V., Cui, C., Zhang, Y., Wang, J., Li, Q., 2009. Protein science: a publication of the Protein Society 18 (5), 936-48.

Spinelli, S., Ramoni, R., Grolli, S., Bonicel, J., Cambillau, C., Tegoni, M.,Taglio, V., 1998. Biochemistry 37 (22), 2960 (98), 7913-7918. 
Spitzer, D., Cottineau, T., Piazzon, N., Josset, S., Schnell, F., Pronkin, S. N., Keller, V., 2012.

Angewandte Chemie (International ed. in English) 51 (22), 5334-8.

Vančura, C., Dufour, I., Heinrich, S. M., Josse, F., Hierlemann, A., 2008. Sensors and Actuators A: Physical141 (1), 43-51.

Vincent, F., Spinelli, S., Ramoni, R., Grolli, S., Pelosi, P., Cambillau, C., Tegoni, M., 2000. Journal of molecular biology 300 (1), 127-39.

Vincent, F., Ramoni, R., Spinelli, S., Grolli, S., Tegoni, M., Cambillau, C., 2004. European journal of biochemistry / FEBS 271 (19), 3832-42.

Wang, Q., Kromka, A., Houdkova, J., Babchenko, O., Rezek, B., Li, M., Szunerits, S., 2012. Langmuir: the ACS journal of surfaces and colloids 28 (1), 587-92.

Wei, Y., Brandazza, A., Pelosi, P., 2008. Biochimica et biophysica acta 1784 (4), 666-71. 